\title{
Produção e colaboração científica em Organização e Representação do Conhecimento: análise bibliométrica do GT2 do ENANCIB no período de 2009 a 2014
}

\author{
Roberta Cristina Dal' Evedove Tartarotti \\ Doutoranda; Universidade Estadual Paulista (UNESP), São Paulo, SP, Brasil; \\ roberta_tartarotti@yahoo.com.br \\ Mariângela Spotti Lopes Fujita \\ Doutora, Universidade Estadual Paulista (UNESP), São Paulo, SP, Brasil; \\ mariangelalf57@gmail.com
}

Resumo: Objetivou-se caracterizar a comunidade científica brasileira em Organização e Representação do Conhecimento da Ciência da Informação, a partir da publicação de trabalhos no GT2 do ENANCIB (Encontro Nacional de Pesquisa em Ciência da Informação) no período de 2009 a 2014 por meio de indicadores bibliométricos de produção e ligação. A partir dos 215 trabalhos apresentados, foram analisados os autores mais produtivos, relações de gênero entre autores, tipo de autoria, instituições mais produtivas, temáticas mais estudadas e redes de colaboração entre autores/instituições, construídas por meio dos softwares Excel e UCINET. Os resultados indicam 27 pesquisadores e 14 instituições como os mais produtivos, com destaque para a Região Sudeste. A maior parte dos trabalhos foi realizada por autores do sexo feminino e em colaboração científica, com predominância de colaboração intrainstitucional, sendo a maior rede de colaboração composta por 37 autores. Houve maior concentração nas abordagens tecnológica e epistemológica e poucos trabalhos em torno da abordagem cultural do campo da Organização e Representação do Conhecimento neste universo de pesquisa.

Palavras-chave: Organização e Representação do Conhecimento. Produção científica. Estudos bibliométricos. Colaboração científica. ENANCIB.

\section{Introdução}

Considerada o fenômeno cultural e social mais importante da contemporaneidade (MALTRÁS BARBA, 2003, p. 11), a ciência pode ser definida como a prática sistemática do "conhecimento que inclua, em qualquer 
forma ou medida, uma garantia da própria validade." (ABBAGNANO, 2000, p. 136). Estabelecida como uma atividade de natureza colaborativa e social, o ideal clássico da ciência é garantir esta validade, explicando a verdade sobre a realidade material do mundo que nos cerca. Composta por distintas ciências em um processo de integração e inter-relação de alta complexidade (CURRÁS, 2009, p. 74), a ciência está normalmente associada à publicação de artigos, "teses, livros, tratados, etc. e seus resultados, ou seja, os conhecimentos por ela gerados são livremente veiculados, por serem considerados patrimônios da civilização, e não objetos de propriedade particular." (TEIXEIRA, 1983, p. 51). Desse modo, "umas das características da ciência é a publicação de seus resultados" (SPINAK, 1998, p. 142).

Nascida mais próxima do contexto contemporâneo, garantindo assim maior "flexibilidade" e "tolerância" relativa à sua consolidação científica (SIQUEIRA, 2010, p. 64), a Ciência da Informação vem se institucionalizando enquanto campo científico, caracterizada por seus objetos, métodos, técnicas, práticas, atores e, mais especificamente, por sua produção científica e avaliação pelos pares.

No Brasil, a Associação Nacional de Pesquisa e Pós-Graduação em Ciência da Informação (ANCIB) é referência na área. Fundada em 1989, reunindo os Programas de Pós-Graduação em Ciência da Informação, docentes, pesquisadores, estudantes de pós-graduação e profissionais de áreas correlatas. Segundo seu Estatuto, o objetivo principal da ANCIB é "promover o desenvolvimento da pesquisa e de estudos avançados em Ciência da Informação no país", incentivando o desenvolvimento de atividades de produção cientifica no ENANCIB - Encontro Nacional de Pesquisa em Ciência da Informação, realizado desde 1994 (ASSOCIAÇÃO NACIONAL DE PESQUISA E PÓSGRADUAÇÃO EM CIÊNCIA DA INFORMAÇÃO, 2010, p. 1).

De acordo com a ANCIB, até o ano de 2016 terão sido realizadas 17 edições do evento, possibilitando "identificar o estado da arte da área, suas frentes de pesquisa, temas de interesse e lacunas de pesquisa a serem preenchidas". No ENANCIB, os Grupos de Trabalho (GT) "constituem espaços para reunião, discussão e intercâmbio entre os pesquisadores", permitindo a 
"interlocução e o compartilhamento de resultados de pesquisas, criando oportunidades de debates e estímulo à reflexão”. Assim, os 11 GT constituem a estrutura da comunicação científica dos Encontros Nacionais de Pesquisa e PósGraduação em Ciência da Informação (ASSOCIAÇÃO NACIONAL DE PESQUISA E PÓS-GRADUAÇÃO EM CIÊNCIA DA INFORMAÇÃO, 2015, não paginado).

Estruturalmente, cada GT é organizado de acordo com temas de pesquisa e metodologias correlatas do campo da Ciência da Informação, correspondendo "a uma clivagem, simultaneamente conceitual e prática" em determinado momento (Norma para funcionamento dos GT). Neste contexto, destaca-se o GT2 - Organização e Representação do Conhecimento, que tem como ementa:

Teorias, metodologias e práticas relacionadas à organização e
preservação de documentos e da informação, enquanto
conhecimento registrado e socializado, em ambiências
informacionais tais como: arquivos, museus, bibliotecas e
congêneres. Compreende, também, os estudos relacionados aos
processos, produtos e instrumentos de representação do
conhecimento (aqui incluindo o uso das tecnologias da informação)
e as relações inter e transdisciplinares neles verificadas, além de
aspectos relacionados às políticas de organização e preservação da
memória institucional. (ASSOCIAÇÂA NACIONAL DE
PESQUISA E PÓS-GRADUAÇÃO EM CIÊNCIA DA
INFORMAÇÃO, 2015, não paginado).

A temática do GT2 refere-se ao domínio $^{1}$ da Organização e Representação do Conhecimento, considerado por Smiraglia (2011) um subdomínio-chave da Ciência da Informação, que é dedicado à ordem conceitual do conhecimento. Para Dahlberg (2014, p. 328), uma

subdisciplina da ciência, com campos de aplicação não somente na Ciência da Informação, mas também para todos os campos de assunto (domínios) que necessitam de taxonomias (sistemas de classificação de objetos).

Historicamente, ao longo dos encontros desde 1994, o GT2 do ENANCIB foi assim denominado: Representação do Conhecimento/Indexação/Teoria da Classificação (1994 a 2003), Organização do Conhecimento e Representação da Informação (2005-2006) e Organização e Representação do Conhecimento (2007 a 2015). 
Diante da relevância do GT2 do ENANCIB na representatividade da Organização e Representação do Conhecimento da Ciência da Informação e da importância dos estudos bibliométricos na ciência para a avaliação de sua produção científica, o objetivo da pesquisa foi: caracterizar a comunidade científica brasileira na temática "Organização e Representação do Conhecimento" a partir da publicação de trabalhos (comunicações orais e pôsteres) no GT2 do ENANCIB no período de 2009 a 2014 por meio de indicadores bibliométricos de produção e ligação. Justifica-se ainda o período de análise em complementação à pesquisa de Oliveira, Grácio e Silva (2010), que realizaram um estudo dos pesquisadores de maior visibilidade na temática a partir da análise de cocitações entre 2003 e 2008.

\section{A abordagem bibliométrica na ciência: da produção à colaboração}

A ciência pode ser definida como um campo social, cujas práticas estão orientadas para a aquisição de autoridade científica. Desse modo, a estrutura do campo científico é definida pelo estado das relações de força entre os atores que nele atuam, onde a posição ocupada por cada ator em um determinado momento "é a resultante, objetivada nas instituições e incorporada nas disposições, do conjunto de estratégias anteriores desse agente e de seus concorrentes" (BOURDIEU, 1983, p. 124; 133-134).

\footnotetext{
A ciência é uma atividade eminentemente coletiva e social, construída por meio de relações sociais entre os pares. Seus resultados (avanços ou retrocessos) são resultantes da interação entre os pesquisadores, seja de maneira direta (por meio de desenvolvimento de projetos e publicações em coautoria) ou indireta (por meio de citação de trabalhos da comunidade científica). De certo modo, existe uma relação entre todos os membros de uma comunidade científica de determinada área, tanto nacionalmente como internacionalmente, e, em consequência, ao desenvolvimento da atividade científica como um todo. (FUJINO et al., 2009, p. 215).
}

A comunicação científica torna-se parte fundamental das práticas do campo científico, ocupando papel central para a produção do conhecimento científico ao materializar e divulgar esse conhecimento tanto para seus pares 
como para a sociedade em geral. Para Meadows (1999, p. 161), "a realização de pesquisas e a comunicação de seus resultados são atividades inseparáveis”.

A comunicação da produção científica é um dos mecanismos mais eficazes no compartilhamento das experiências e atividade indispensável para alimentação do fluxo que leva ao desenvolvimento da ciência. É a expressão dos saberes de um determinado campo de conhecimento que se torna insumo para compreender a história da própria ciência, refletir sobre ela, avaliá-la e identificar novos caminhos de pesquisa. (FUJINO et al., 2009, p. 216).

Por definição, a Bibliometria "é o estudo dos aspectos quantitativos da produção, disseminação e uso da informação registrada", desenvolvendo "padrões e modelos matemáticos para medir esses processos, usando seus resultados para elaborar previsões e apoiar a tomada de decisões." (MACIASCHAPULA, 1998, p. 134). Em outra definição, é “o conjunto de conhecimentos metodológicos para a aplicação de técnicas quantitativas, destinadas ao estudo dos processos de produção, comunicação e uso da informação científica", cujo objetivo é "contribuir para a análise e avaliação da ciência e da pesquisa." (CARRIZO SAINERO, 2000, p. 9).

Desse modo, por meio de ferramentas, modelos matemáticos, estatísticos e computacionais, é possível realizar análises quantitativas e comparativas das pesquisas científicas. Esses estudos são úteis para mapeamento de um determinado campo científico. Nesse cenário, as abordagens bibliométricas como um referencial metodológico quantitativo são fundamentais, ao utilizar indicadores bibliométricos que evidenciam determinados aspectos da comunicação científica, dependendo dos objetivos determinados. Por meio do uso destes indicadores, "é possível saber como se dá a comunicação cientifica de uma área do conhecimento, obtendo-se assim um 'mapeamento' da mesma”. Além disso, retrata o comportamento e a evolução do conhecimento em um determinado campo científico (VANZ; CAREGNATO, 2003, p. 251).

Como apontam Marcelo e Hayashi (2013, p. 221), embora a maior aplicação seja no campo da Ciência da Informação, os estudos bibliométricos podem ser utilizados em todas as áreas do conhecimento, "a fim de explorar o impacto da produção de um determinado campo de conhecimento, a produção e 
produtividade de um conjunto de investigadores, por meio da construção de indicadores bibliométricos". Dependendo dos objetivos, enfoques e aplicações, são diversos os indicadores bibliométricos, dentre os quais se destacam os indicadores de produção científica, os indicadores de citação e os indicadores de colaboração científica (OKUBO, 1997; SPINAK, 1998).

Um aspecto importante do comportamento científico é a colaboração entre os pares, seja a nível individual, institucional ou até mesmo entre países. Segundo Katz e Martin (1997, p. 7), a colaboração científica pode ser definida como "o trabalho conjunto de pesquisadores para atingir um objetivo comum de produzir novos conhecimentos científicos", sendo a coautoria um indicador de colaboração científica. O colaborador pode ser qualquer ator que contribui em parte de uma pesquisa. Uma das características da ciência na contemporaneidade é a colaboração, mais forte em determinadas áreas científicas do que outras, mas sempre presente, confirmando "a importância de colaboração na ciência para difundir a informação, podendo ser entre pesquisadores da mesma instituição, entre pesquisadores de outras universidades bem como entre instituições de pesquisas nacionais e internacionais" (FUNARO et al., 2009, p. 362).

A interação entre os atores no campo científico caracteriza-se como uma rede social, definida como um conjunto "de nós e laços com relações ilimitadas e hibridas articuladas entre sujeitos, objetos e discursos, que interagem no mundo real e no virtual" (POBLACIÓN; MUGNAINI; RAMOS, 2009, p. 626) ou "de pontos (atores sociais) vinculados por uma série de relações que cumprem determinadas propriedades" (CURRÁS, 2009, p. 61).

Uma rede social passa a ser denominada de rede de colaboração ou rede de coautoria científica quando há integração entre os atores no intuito de partilharem a publicação de trabalhos científicos, ou seja, “constituída por pessoas que se relacionam dentro de um contexto de pesquisa acadêmica formando nós de informação que geram a colaboração entre pesquisadores" (POBLACIÓN; MUGNAINI; RAMOS, 2009, p. 627). Desse modo, as redes de pesquisadores são consideradas fundamentais para o desenvolvimento da ciência em geral.

Por possuir uma estrutura característica, a análise das redes sociais científicas permite quantificar as relações e seu consequente tratamento 
matemático, evidenciando importantes aplicações para a análise e interpretação do comportamento social (CURRÁS, 2009, p. 61). Assim, por meio da análise das redes de colaboração científica, é possível identificar o comportamento dos atores e grupos de atores que ocupam determinadas posições na rede.

Além destes apontamentos, a "produção de trabalhos em colaboração institucional constitui uma rica possibilidade de compartilhamento e socialização do conhecimento e informações em um determinado contexto científico", e as "pesquisas destinadas à análise da produção científica são relevantes na Ciência da Informação na medida em que estabelecem os caminhos percorridos pela comunidade científica e apresentam as características específicas de cada área e temática” (DAL' EVEDOVE; FUJITA; TARTAROTTI, 2013, p. 15).

\section{Procedimentos metodológicos}

A pesquisa se caracteriza como exploratória e descritiva. A primeira fase metodológica consistiu no levantamento dos dados a partir de súmula dos 215 trabalhos apresentados, disponíveis em formato eletrônico no Repositório Questões em Rede ${ }^{2}$, na Coleção BENANCIB, onde constam o título da pesquisa, autor(es), filiação institucional, resumo, palavras-chave e referências bibliográficas. O universo constituiu-se de 29 trabalhos apresentados no X ENANCIB, em 2009 (João Pessoa); 29 no XI ENANCIB (Rio de Janeiro) em 2010; 35 no XII ENANCIB (Brasília) em 2011; 28 trabalhos no XIII ENANCIB (Rio de Janeiro) em 2012; 40 no XIV ENANCIB em 2013 (Florianópolis) e 54 no XV ENANCIB (Belo Horizonte) em 2014.

Cabe ressaltar que os Anais referentes a 2011 e 2014 não apresentavam a filiação dos autores, sendo pesquisadas posteriormente no Currículo Lattes dos mesmos, considerando-se a instituição em que o autor estava vinculado no ano da publicação do artigo.

Entre 2009 e 2014 foram publicados 215 trabalhos no GT2 do ENANCIB, com 443 autorias e $\mathbf{2 6 3}$ pesquisadores, perfazendo uma média de 36 trabalhos por ano. Para a análise dos dados, inicialmente, foram construídos seis 
arquivos, cada um com o conjunto de publicações realizado em cada ano. Em seguida, foram gerados novos arquivos, a saber: arquivo contendo o rol da totalidade das autorias; arquivo contendo o rol de autores participantes no GT2 do ENANCIB, no período estudado; arquivo contendo o rol da totalidade de autorias institucionais por trabalho; arquivo contendo o rol das temáticas mais abordadas; arquivo contendo o rol das referências bibliográficas de todos os trabalhos.

A segunda fase metodológica consistiu na aplicação dos procedimentos bibliométricos, onde foram analisadas as seguintes variáveis: Autores: autores mais produtivos; relações de gênero entre os autores; tipo de autoria (simples ou múltipla); rede de colaboração/coautoria entre os autores; Autores institucionais: instituições mais produtivas; rede de colaboração/coautoria entre as instituições; Temáticas: temáticas mais estudadas.

Em relação aos indicadores relacionados aos Autores, do total de $\mathbf{2 6 3}$ pesquisadores encontrados, foram considerados os mais produtivos aqueles que publicaram pelo menos quatro trabalhos no GT2 do ENANCIB no período analisado. No tocante aos indicadores relacionados aos Autores institucionais, das 40 instituições participantes, foram consideradas as mais produtivas aquelas que apresentaram pelo menos cinco trabalhos no período estudado. Já em relação às Temáticas mais abordadas no corpus analisado, considerando-se a totalidade dos 215 trabalhos, as temáticas mais relevantes foram retiradas a partir das palavras-chave, sendo que o resumo foi utilizado quando os trabalhos não apresentavam as mesmas (quatro no total). Partiu-se do pressuposto de que a análise destes elementos são indicadores das questões mais estudadas e, portanto, aquelas que mais constituem foco de pesquisas no GT2 do ENANCIB.

Para análise de coautoria institucional foram consideradas aquelas que tiveram pelo menos um trabalho em coautoria, seja interinstitucional ou intrainstitucional, totalizando 40 instituições com este perfil. Dessas, 37 instituições desenvolveram pelo menos um trabalho em parceria com outras instituições, ou seja, somente 3 realizaram trabalhos apenas em colaboração interna.

Partindo-se das relações estabelecidas socialmente, foram desenvolvidos 
"programas e softwares que interpretam estas interações, através de teorias das estruturas na matemática e na estatística aplicadas à informática, sempre com uma categorização de trabalhar as informações em frações cartesianas." Por meio da visualização dos laços fortes e frágeis da rede, é possível visualizar "uma representação de poder através da frequência e da intensidade com que se mostram as relações" na produção e comunicação científica (PINTO et al., 2009, p. 297).

Desse modo, para a elaboração da rede de colaboração entre os autores, construiu-se uma matriz quadrada (simétrica) de 149x149, isto é, com 149 autores e seus relacionamentos no software Excel (Figura 1). Os nomes dos autores foram padronizados, utilizando-se primeiro o sobrenome e os demais nomes de forma abreviada:

Figura 1 - Recorte da matriz quadrada 149x149 para elaboração da rede de coautoria.

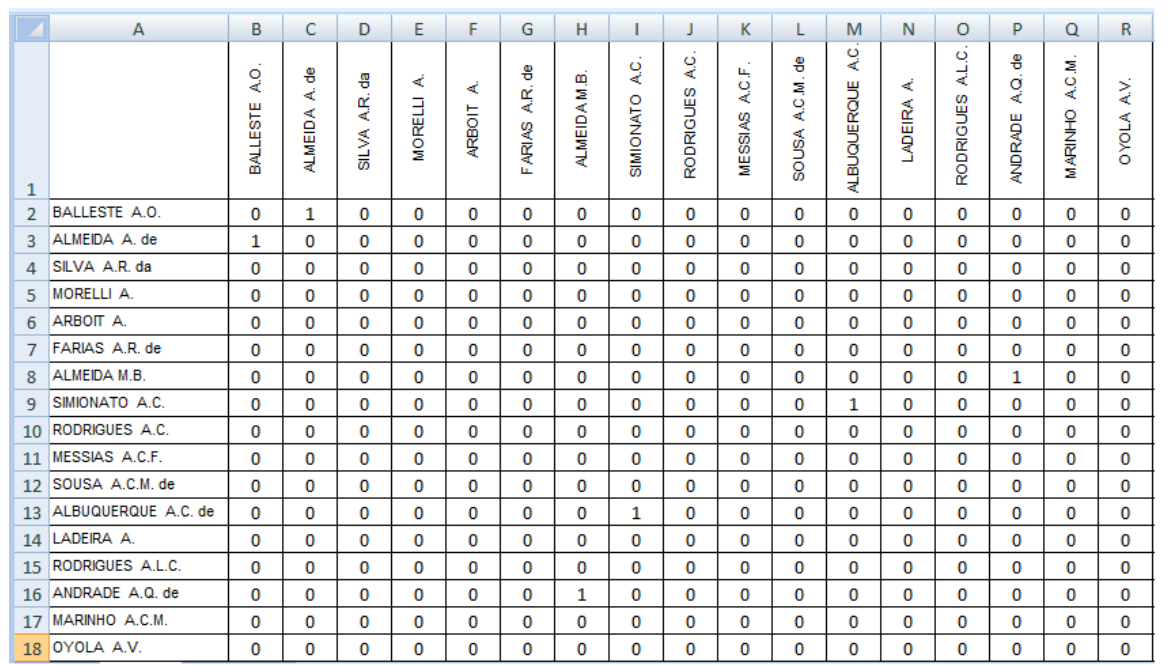

Fonte: Dados da pesquisa.

Para a elaboração da rede de colaboração institucional, construiu-se uma matriz quadrada 40x40 (ou seja, 40 instituições) ainda no programa Excel (Figura 2): 
Figura 2 - Recorte da matriz quadrada 40x40 para elaboração da rede de colaboração institucional.

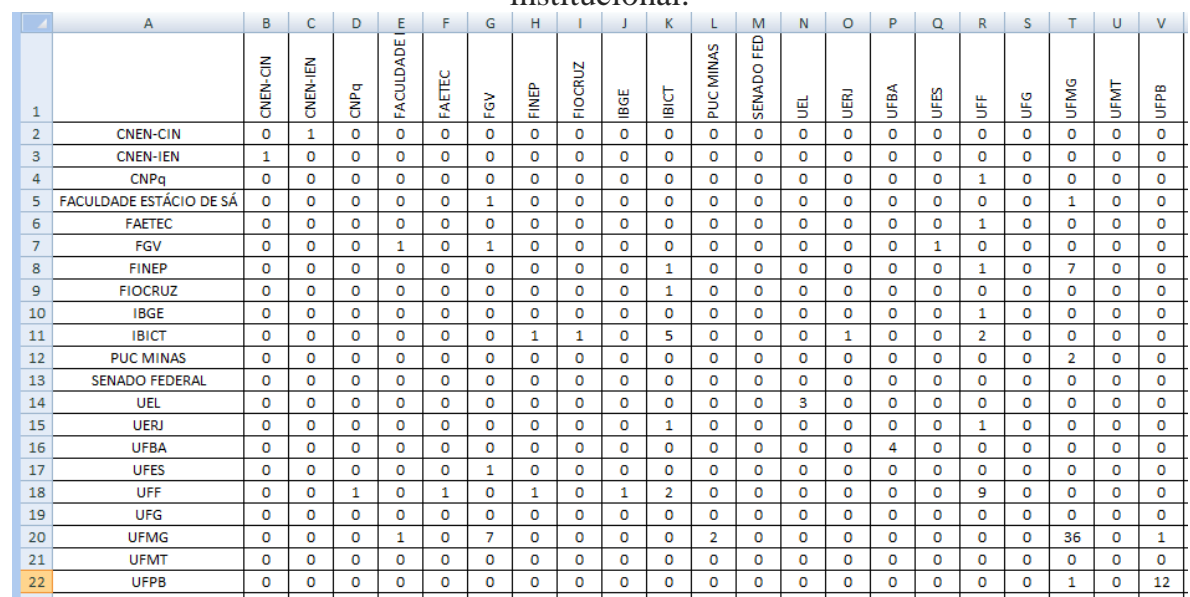

Fonte: Dados da pesquisa.

Em ambas as matrizes, o valor de cada ocorrência foi registrado, utilizando-se a opção do software Excel "Localizar e selecionar", "Ir para" “especial”, "em branco", para o preenchimento de todas as células em branco na matriz com zeros. Por meio das matrizes padronizadas, foi possível então gerar a representação gráfica das redes no software UCINET, versão 6.29, disponível gratuitamente para download na internet. Faz parte do pacote UCINET o software NetDraw, que possibilita a visualização das redes em duas dimensões.

\section{Apresentação e análise dos dados}

De forma geral, por meio do levantamento e análise bibliométrica dos dados, foi possível verificar um total de 215 trabalhos e 263 autores participantes. Para a análise das relações de gênero existentes entre os autores que publicaram no GT2 do ENANCIB entre 2009 e 2014, utilizou-se o número de autorias. Das 443 encontradas, identifica-se no Gráfico 1 a predominância de trabalhos escritos por autores do sexo feminino, perfazendo um total de $66 \%$. As autorias do sexo masculino correspondem a 34\%. A predominância do sexo feminino teve maior incidência em 2013, com 70\%, e em 2010, com 68\%. A maior equidade entre os gêneros foi observada no ano 2009, quando a diferença foi da ordem de apenas $8 \%$. 
Produção e colaboração científica em Organização e Representação do Conhecimento: análise bibliométrica do GT2 do ENANCIB no período de 2009 a 2014

Roberta Cristina Dal'Evedove Tartarotti, Mariângela Spotti Lopes Fujita

Tabela 1 - Tendência dos gêneros das autorias.

\begin{tabular}{c|c|c|c|c|c}
\hline ANO & HOMEM & Fi & MULHER & Fi & N. AUTORIAS \\
\hline 2009 & 22 & $41 \%$ & 31 & $49 \%$ & 53 \\
\hline 2010 & 20 & $32 \%$ & 43 & $68 \%$ & 63 \\
\hline 2011 & 26 & $37 \%$ & 45 & $63 \%$ & 71 \\
\hline 2012 & 19 & $37 \%$ & 33 & $63 \%$ & 52 \\
\hline 2013 & 27 & $30 \%$ & 62 & $70 \%$ & 89 \\
\hline 2014 & 38 & $33 \%$ & 77 & $67 \%$ & 115 \\
\hline TOTAL & $\mathbf{1 5 2}$ & $\mathbf{3 4 \%}$ & $\mathbf{2 9 1}$ & $\mathbf{6 6 \%}$ & $\mathbf{4 4 3}$ \\
\hline
\end{tabular}

.Nota: Fi = Frequência. Fonte: Dados da pesquisa.

Gráfico 1 - Tendência dos gêneros das autorias.

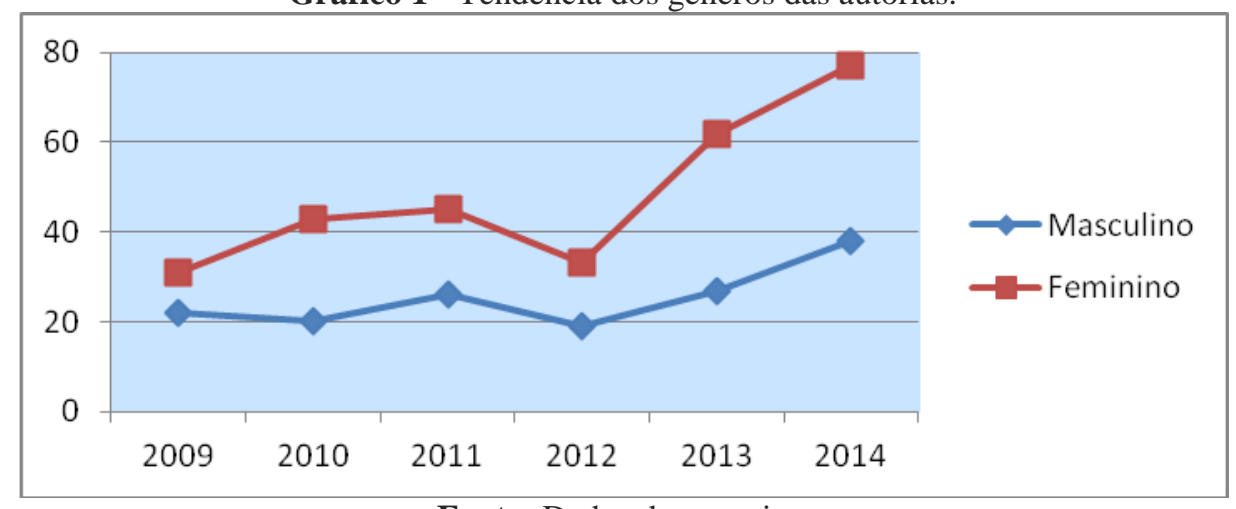

Fonte: Dados da pesquisa.

Para o levantamento dos autores mais produtivos no GT2 do ENANCIB, os autores que compuseram o corpus do estudo foram codificados e apresentados por ordem de produtividade no período abarcado, cujos nomes foram abreviados, destacando-se os sobrenomes e indicando a instituição de origem. Ademais, identificou-se o número de trabalhos por ano, o total de trabalhos por autor, bem como o número de coautores com que cada um firmou parcerias de pesquisa, cujos dados são apresentados na Tabela 2, a saber:

Tabela 2 - Autores mais produtivos.

\begin{tabular}{|c|c|c|c|c|c|c|c|c|c|}
\hline \multirow{2}{*}{ ORDEM } & \multirow{2}{*}{ AUTORES } & \multicolumn{7}{|c|}{ ANO DE PUBLICAÇÃO } & \multirow{2}{*}{$\begin{array}{c}\text { N. DE } \\
\text { COAUTORES }\end{array}$} \\
\hline & & 2009 & 2010 & 2011 & 2012 & 2013 & 2014 & Total & \\
\hline $1^{\mathrm{o}}$ & $\begin{array}{l}\text { CAMPOS, M. L. de } \\
\text { A. (UFF) }\end{array}$ & 1 & 2 & 3 & 2 & 2 & 2 & 12 & 10 \\
\hline $2^{\circ}$ & $\begin{array}{llll}\begin{array}{l}\text { SOUZA, } \\
\text { (IBICT) }\end{array} & \text { R. F. de } \\
\end{array}$ & - & 2 & 2 & 3 & 2 & 2 & 11 & 13 \\
\hline $3^{\circ}$ & SOUZA, R. R. (FGV) & 1 & 2 & 1 & - & 3 & 2 & 9 & 21 \\
\hline $4^{\circ}$ & $\begin{array}{l}\begin{array}{l}\text { ALMEIDA, } \\
\text { (UFMG) }\end{array} \text { M. B. } \\
\end{array}$ & 1 & - & 3 & 1 & 1 & 2 & 8 & 13 \\
\hline $5^{\circ}$ & $\begin{array}{l}\text { LIMA, G. A. B. de O. } \\
\text { (UFMG) }\end{array}$ & 1 & 1 & 2 & 1 & - & 3 & 8 & 5 \\
\hline $6^{\circ}$ & $\begin{array}{l}\text { MOURA, } \\
\text { (UFMG) }\end{array}$ & - & 1 & 2 & 1 & 1 & 2 & 7 & 7 \\
\hline
\end{tabular}




\begin{tabular}{|c|c|c|c|c|c|c|c|c|c|}
\hline $7^{\circ}$ & $\begin{array}{l}\text { NEVES, D. A. de B. } \\
\text { (UFPB) }\end{array}$ & 1 & 3 & 1 & 1 & - & 1 & 6 & 4 \\
\hline $8^{\circ}$ & $\begin{array}{l}\text { MORAES, J. B. E. de } \\
\text { (UNESP) }\end{array}$ & 2 & 1 & - & 1 & 1 & 1 & 6 & 4 \\
\hline $9^{\circ}$ & $\begin{array}{ll}\begin{array}{l}\text { ALVARENGA, } \\
\text { (UFMG) }\end{array} & \text { L. } \\
\end{array}$ & 2 & 1 & - & 1 & 1 & 1 & 6 & 7 \\
\hline $10^{\circ}$ & $\begin{array}{l}\text { MADIO, T. C. de C. } \\
\text { (UNESP) }\end{array}$ & - & - & 2 & 1 & 2 & 1 & 6 & 5 \\
\hline $11^{\circ}$ & $\begin{array}{l}\text { RODRIGUES, A. C. } \\
\text { (UFF) }\end{array}$ & 1 & - & 1 & 2 & - & 2 & 6 & 2 \\
\hline $12^{\circ}$ & $\begin{array}{l}\text { ALBUQUERQUE, } \\
\text { A. C. de (UEL) }\end{array}$ & 1 & 1 & - & 1 & 1 & 1 & 5 & 3 \\
\hline $13^{\circ}$ & $\begin{array}{lll}\begin{array}{l}\text { CAMPOS, } \\
\text { (UFRJ) }\end{array} & \text { L. } & \text { M. } \\
\end{array}$ & - & 1 & 1 & 2 & - & 1 & 5 & 3 \\
\hline $14^{\circ}$ & $\begin{array}{l}\text { GUIMARÃES, J. A. } \\
\text { C. (UNESP) }\end{array}$ & - & 1 & 2 & - & 1 & 1 & 5 & 6 \\
\hline $15^{\circ}$ & $\begin{array}{lll}\begin{array}{l}\text { KOBASHI, } \\
(\text { USP) }\end{array} & \text { N. } & \text { Y. } \\
\end{array}$ & 1 & 1 & 1 & 1 & 1 & - & 5 & 3 \\
\hline $16^{\circ}$ & $\begin{array}{l}\text { LARA, M. L. G. de } \\
\text { (USP) }\end{array}$ & 1 & - & 2 & - & 1 & 1 & 5 & 5 \\
\hline $17^{\circ}$ & $\begin{array}{lll}\begin{array}{l}\text { MANINI, } \\
\text { (UNB) }\end{array} & \text { M. } & \text { P. } \\
\end{array}$ & 1 & 2 & 1 & - & 1 & - & 5 & 3 \\
\hline $18^{\circ}$ & $\begin{array}{l}\text { SILVA, M. B. da } \\
\text { (UFBA) }\end{array}$ & - & 1 & 1 & 1 & 1 & 1 & 5 & 2 \\
\hline $19^{\circ}$ & $\begin{array}{l}\text { ALBUQUERQUE, } \\
\text { M. E. B. C. de (UFPB) }\end{array}$ & - & - & 1 & - & 1 & 2 & 4 & 3 \\
\hline $20^{\circ}$ & $\begin{array}{l}\text { CERVANTES, B. M. } \\
\text { N. (UEL) }\end{array}$ & - & 1 & 1 & 2 & - & - & 4 & 4 \\
\hline $21^{\circ}$ & $\begin{array}{lll}\begin{array}{l}\text { CORREA, } \\
\text { (UFPE) }\end{array} & \text { R. } & \text { F. } \\
\end{array}$ & - & - & - & 1 & 2 & 1 & 4 & 3 \\
\hline $22^{\circ}$ & $\begin{array}{l}\text { FUJITA, M. } \quad \text { S. L. } \\
\text { (UNESP) }\end{array}$ & - & - & - & - & 1 & 3 & 4 & 4 \\
\hline $23^{\circ}$ & $\begin{array}{l}\text { MARCONDES, C. H. } \\
\text { (UFF) }\end{array}$ & 1 & 1 & - & - & 1 & 1 & 4 & 3 \\
\hline $24^{\circ}$ & $\begin{array}{l}\text { MENDONÇA, F. M. } \\
\text { (UFMG) }\end{array}$ & - & - & 1 & 1 & 1 & 1 & 4 & 3 \\
\hline $25^{\circ}$ & $\begin{array}{l}\text { MOREIRA, } \\
\text { (UNESP) }\end{array}$ & - & - & 1 & - & 2 & 1 & 4 & 5 \\
\hline $26^{\circ}$ & PINHO, F. A. (UFPE) & - & - & 1 & - & 2 & 1 & 4 & 4 \\
\hline $27^{\circ}$ & $\begin{array}{l}\text { SOUZA, J. C. C. E. de } \\
\text { (UFF) }\end{array}$ & - & - & 2 & - & 1 & 1 & 4 & 2 \\
\hline Total & 27 & 15 & 22 & 32 & 23 & 31 & 35 & 157 & 147 \\
\hline
\end{tabular}

De acordo com os dados apresentados, os 27 autores mais produtivos publicaram 157 trabalhos, entre comunicações orais e pôsteres, o que representa $73 \%$ de toda a produção científica do período analisado. A autora mais produtiva, ou seja, Campos, M.L. de A. publicou 12 trabalhos no GT2 do ENANCIB entre 2009 e 2014, seguida por Souza, R.F. de com 11 trabalhos, Souza, R.R. de com nove trabalhos e Almeida, M.B. e Lima, G.A.B. de O., com oito trabalhos cada. Com sete trabalhos publicados aparece Moura, M.A. O conjunto de autores que publicaram seis trabalhos é composto por Neves, D.A. de B.; Moraes, J. B. E. de; Alvarenga, L.; Madio, T.C. de C. e Rodrigues, A.C. Outro conjunto formado por Albuquerque, A.C. de; Campos, L.M.; Guimarães, 
J.C.C.; Kobashi, N.Y.; Lara, M.L.G. de; Manini, M.P. e Silva, M.B. da publicaram cinco trabalhos cada. Já com quatro trabalhos aparecem Albuquerque, M.E.B.C. de; Cervantes, B.M.N.; Correa, R.F.; Fujita, M.S.L.; Marcondes, C.H.; Mendonça, F.M.; Moreira, W.; Pinho, F.A. e Souza, J.C.C.E. de.

Em referência às relações estabelecidas com outros autores, dos trabalhos produzidos em coautoria pelo grupo de autores mais produtivos houve colaboração com outros 147 coautores, sendo excluídos, por sua vez, os nomes que se repetiram. Souza, R.R. estabeleceu mais relações de colaboração científica (21), seguido por Souza, R.F. de e Almeida, M.B., respectivamente com 13 autores; Campos, M.L.de A. (10); Moura, M.A. e Alvarenga (7); Guimarães, J.A.C. (6); Lima, G.A.B. de O, Moreira, W. e Madio, T.C.de (5); Neves, D.A.de B., Moraes, J.B.E. de, Cervantes, B.M.N., Fujita, M.S.L. e Pinho, F.A. (4); Albuquerque, A.C. de, Campos, L.M., Kobashi, N.Y., Manini, M.P. Albuquerque, M.E.B.C. de, Marcondes, C.H. (3) e Rodrigues, A.C., Silva, M.B. da e SOUZA, J.C.C.E. de, com duas colaborações cada.

Outra variável levantada foi em relação ao tipo de colaboração científica entre os autores, ou seja, à distribuição dos trabalhos por tipo de autoria e ano, apresentada na Tabela 3:

Tabela 3 - Distribuição dos trabalhos por tipo de autoria e ano.

\begin{tabular}{|c|c|c|c|c|c|c|c|c|c|c|}
\hline \multirow[t]{2}{*}{ ANO } & \multicolumn{2}{|c|}{ 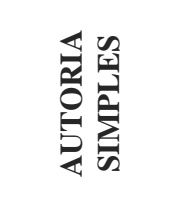 } & \multicolumn{2}{|c|}{ 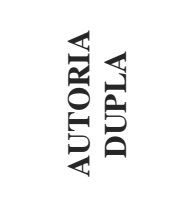 } & \multicolumn{2}{|c|}{ 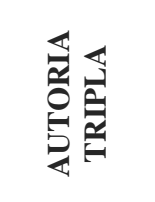 } & \multicolumn{2}{|c|}{ 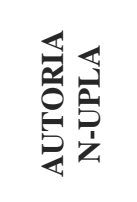 } & \multicolumn{2}{|c|}{ 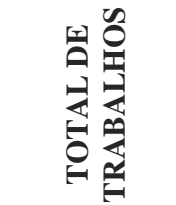 } \\
\hline & $\mathrm{Fi}$ & $\%$ & $\mathrm{Fi}$ & $\%$ & $\mathrm{Fi}$ & $\%$ & $\mathrm{Fi}$ & $\%$ & $\mathrm{Fi}$ & $\%$ \\
\hline 2009 & 7 & $24 \%$ & 20 & $69 \%$ & 2 & $7 \%$ & - & - & 29 & $13,5 \%$ \\
\hline 2010 & 6 & $21 \%$ & 16 & $55 \%$ & 5 & $17 \%$ & 2 & $7 \%$ & 29 & $13,5 \%$ \\
\hline 2011 & 9 & $26 \%$ & 22 & $63 \%$ & 1 & $3 \%$ & 3 & $9 \%$ & 35 & $16 \%$ \\
\hline 2012 & 8 & $29 \%$ & 17 & $61 \%$ & 2 & $7 \%$ & 1 & $4 \%$ & 28 & $13 \%$ \\
\hline 2013 & 2 & $5 \%$ & 30 & $75 \%$ & 7 & $17 \%$ & 1 & $2 \%$ & 40 & $19 \%$ \\
\hline 2014 & 10 & $18 \%$ & 38 & $70 \%$ & 5 & $9 \%$ & 1 & $2 \%$ & 54 & $25 \%$ \\
\hline Total de Trabalhos & 42 & - & 143 & - & 22 & - & 8 & - & 215 & - \\
\hline PORCENTUAL & - & $19,5 \%$ & - & $66,5 \%$ & - & $10 \%$ & - & $4 \%$ & - & $100 \%$ \\
\hline
\end{tabular}


No tocante ao nível de colaboração científica identifica-se, do grupo de 173 trabalhos publicados em coautoria, que a autoria individual representa $19,5 \%$ dos trabalhos, enquanto que a autoria envolvendo dois atores constitui a forma mais frequente de colaboração e representa 66,5\% dos trabalhos, enquanto a porcentagem de trabalhos escritos por três autores foi de $10 \%$ e de quatro ou mais autores foi de apenas $4 \%$. A quantidade de autores por trabalho variou entre um e quatorze. Os trabalhos escritos em colaboração científica representam 80,5\% do total publicado no período abarcado. Em 2009, $76 \%$ dos trabalhos foram em coautoria, em 2010, 79\%, em 2011, 75\%, em 2012, 72\%, em $2013,94 \%$, e em $2014,81 \%$ foram realizados em colaboração científica, sendo que a autoria dupla teve a maior incidência, com $66,5 \%$ do total publicado no período abarcado.

Para o cálculo do Índice de Colaboração (IC) (SPINAK, 1996), ou seja, para a média ponderada de autores por trabalho, foi utilizada a fórmula $I C=\sum i . N i / N$, Onde: $N=$ total de trabalhos; $i=$ quantidade de autores no trabalho; $\mathrm{Ni}=$ quantidade de trabalhos com $i$ autores. Desse modo, considerando-se a totalidade de 215 trabalhos e 443 autorias, ao aplicarmos os valores identificados na fórmula, obteve-se o índice de colaboração (IC) $=2,06$, isto é, em média, cada trabalho foi produzido por 2,06 autores.

Do conjunto de 173 trabalhos desenvolvidos em cooperação, 130 (75\%) foram escritos em colaboração interna, ou seja, entre autores pertencentes à mesma instituição (colaboração intrainstitucional), enquanto que 31 (18\%) dos trabalhos resultam de colaboração envolvendo autores pertencentes a diferentes instituições (colaboração interinstitucional). Os trabalhos realizados tanto em colaboração intrainstitucional como em colaboração interinstitucional representam $11(6 \%)$ e apenas 1 trabalho (0,6\%) foi desenvolvido em colaboração internacional. De forma geral, verifica-se que a maior incidência de colaboração científica advém de autores pertencentes à mesma instituição de pesquisa, mais especificamente na colaboração entre orientador e orientando de mestrado/doutorado.

Considerando-se o agrupamento de coautorias entre os autores, a perspectiva acerca da concentração das pesquisas do GT2 do ENANCIB entre 
2009 e 2014 pode ser percebida por meio da rede que reflete este aspecto, indicando as relações entre os autores. Os círculos em vermelho correspondem aos autores mais produtivos e os círculos em azul aos demais autores que publicaram os trabalhos em coautoria. A espessura das linhas refere-se à frequência de colaboração, ou seja, quanto mais espessa a linha, maior a quantidade de trabalhos desenvolvidos entre dois determinados autores no período analisado (Figura 3).

Figura 3 - Representação gráfica da rede de colaboração dos autores.

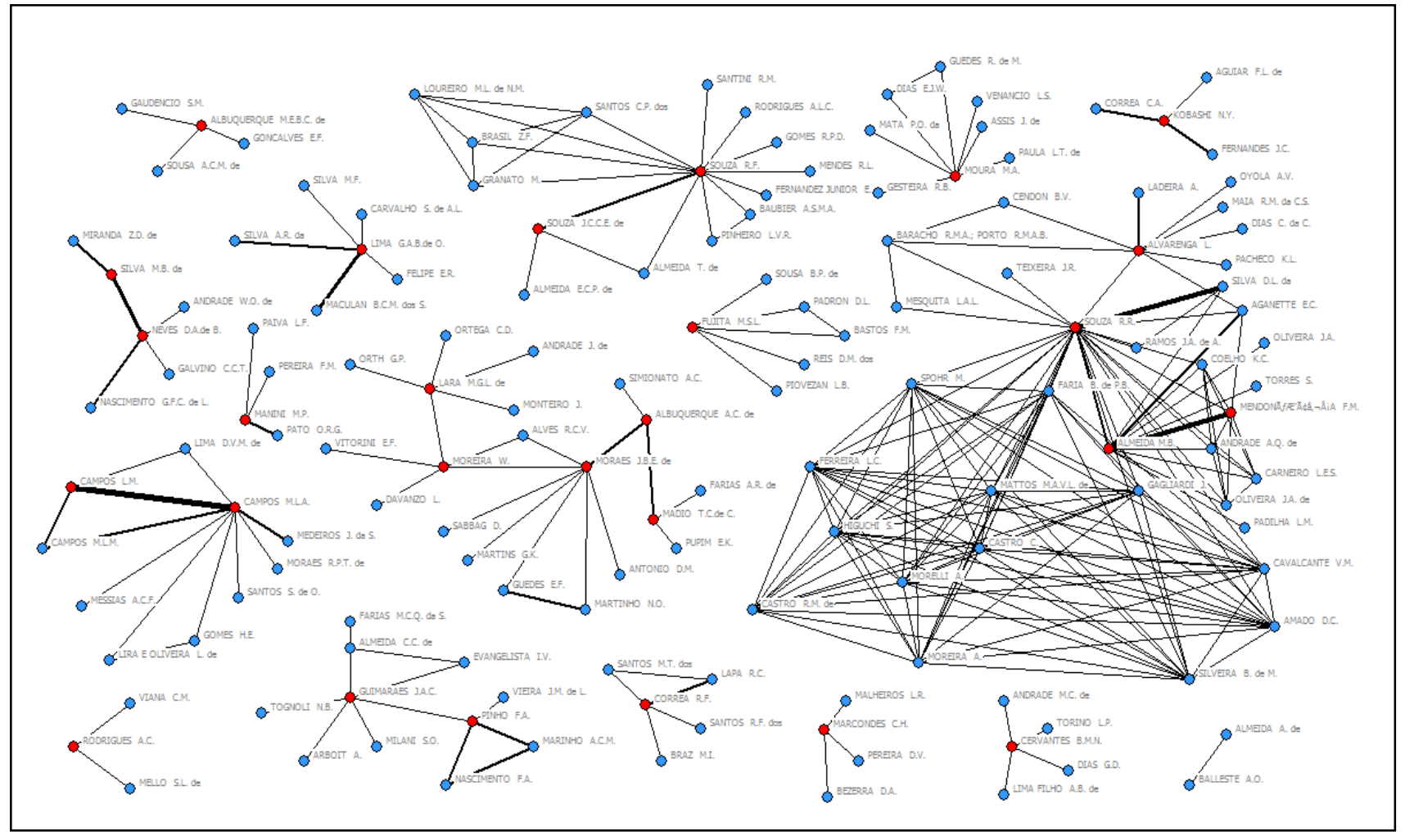

Fonte: Dados da pesquisa.

Ao analisarmos a rede, verifica-se a formação de sub-redes nas quais há um membro central, ou seja, um autor mais produtivo que aglutina os demais autores para uma relação de coautoria.

A maior incidência de relações ocorreu entre Campos, L.M. e Campos, M.L.A., com cinco trabalhos desenvolvidos em colaboração, seguidas por Souza, R.R. com Silva, D.L. e Almeida, M.B. e Mendonça, F.M. com quatro trabalhos cada; Neves, D.A. de B. e Silva, M.B. da; Lima, G.A.B. de O. com 
Maculan, B.C.M. com três trabalhos. As demais relações são da ordem de dois ou um trabalho desenvolvido em coautoria.

Em relação aos autores mais produtivos, a rede de Moraes, J.B.E. de está ligada com a rede de Albuquerque, A.C. de, por sua vez ligada a Madio, T.C. de C. O mesmo autor ainda se conecta à rede de Moreira, W., que se conecta à rede de Lara, M.G.L. de. A rede formada por Silva, M.B. de conecta-se com a rede de Neves, D.A. de B. A rede de Campos, M.L.A. conecta-se com a de Campos, L.M. A rede de Guimarães, J.A.C., composta por cinco autores conecta-se com a rede de Pinho, F.A, que tem relação com três autores. Já a rede de Souza, R.F. composta por 11 autores, conecta-se com Souza, J.C.C.E. de, que possui conexão com um autor isolado e um compartilhado.

Observam-se ainda outras redes de autores mais produtivos, porém isoladas, com a respectiva quantidade de coautores: Moura, M.A. (7); Lima, G.A.B. de O. e Fujita, M.S.L. (5); Cervantes, B.M.N. e Correa, R.F. (4); seguidas por Kobashi, N.Y.; Marcondes, C.H. e Manini, M.P. (3) e Rodrigues, A.C.

A maior rede, ou seja, a com maior número de linhas é composta por 37 autores, participando quatro dos mais produtivos: Souza, R.R.; Almeida, M.B.; Mendonça, F.M. e Alvarenga, L. É a rede com maior número de autores mais produtivos. Observa-se ainda uma díade isolada, composta por Almeida, A. de e Balleste, A.O. e uma tríade, por Viana, C.M.; Mello, S.L. e Rodrigues, A.C.

Para o levantamento das instituições participantes, a Tabela 4 apresenta o rol das instituições mais produtivas, segundo o critério estabelecido inicialmente, ou seja, aquelas cujos autores publicaram pelo menos cinco trabalhos no período analisado.

Tabela 4 - Instituições mais produtivas.

\begin{tabular}{c|c|c|c|c|c|c|c|c|c}
\hline \multirow{2}{*}{ ORDEM } & \multirow{2}{*}{ INSTITUIÇÃo } & \multicolumn{7}{|c}{ ANO DE PUBLICAÇÃO } \\
\cline { 3 - 10 } & & 2009 & 2010 & 2011 & 2012 & 2013 & 2014 & $\begin{array}{c}\text { Total de } \\
\text { participação }\end{array}$ & Fi \\
\hline $1^{\mathbf{0}}$ & UFMG & 8 & 7 & 7 & 6 & 8 & 13 & $\mathbf{4 9}$ & $21 \%$ \\
\hline $2^{\mathbf{0}}$ & UNESP & 7 & 3 & 5 & 4 & 8 & 9 & $\mathbf{3 6}$ & $16 \%$ \\
\hline $3^{\mathbf{0}}$ & UFF & 3 & 4 & 7 & 6 & 6 & 7 & $\mathbf{3 3}$ & $14 \%$ \\
\hline $4^{\mathbf{0}}$ & USP & 3 & 1 & 4 & 1 & 3 & 2 & $\mathbf{1 4}$ & $6 \%$ \\
\hline $5^{\mathbf{0}}$ & UFPB & 3 & 3 & 2 & 1 & 1 & 4 & $\mathbf{1 4}$ & $6 \%$ \\
\hline $6^{\mathbf{0}}$ & UNB & 3 & 4 & 2 & 1 & 2 & 2 & $\mathbf{1 4}$ & $6 \%$ \\
\hline $7^{\mathbf{0}}$ & IBICT & - & 1 & 3 & 4 & 2 & 3 & $\mathbf{1 3}$ & $6 \%$ \\
\hline $8^{\mathbf{o}}$ & UFRJ & 1 & 2 & 1 & 4 & - & 3 & $\mathbf{1 1}$ & $5 \%$ \\
\hline
\end{tabular}


Roberta Cristina Dal' Evedove Tartarotti, Mariângela Spotti Lopes Fujita

\begin{tabular}{c|c|c|c|c|c|c|c|c|c}
\hline $9^{\circ}$ & UFPE & - & - & 1 & 1 & 5 & 3 & $\mathbf{1 0}$ & $4 \%$ \\
\hline $10^{\circ}$ & FGV & 1 & 2 & 1 & - & 3 & 2 & $\mathbf{9}$ & $4 \%$ \\
\hline $11^{\circ}$ & UNIRIO & 1 & - & 1 & 1 & 3 & 2 & $\mathbf{8}$ & $3 \%$ \\
\hline $12^{\mathbf{o}}$ & UEL & - & 1 & - & 3 & 1 & 1 & $\mathbf{6}$ & $3 \%$ \\
\hline $13^{\circ}$ & UFBA & 1 & 1 & 1 & - & 1 & 1 & $\mathbf{5}$ & $2 \%$ \\
\hline $14^{\circ}$ & UFSC & - & - & 1 & - & 2 & 2 & $\mathbf{5}$ & $2 \%$ \\
\hline
\end{tabular}

Fonte: Dados da pesquisa.

A análise da tabela 4 mostra que, do total de $\mathbf{4 0}$ instituições identificadas, 14 (35\%) são as mais produtivas e participam em $\mathbf{4 3 , 5 \%}$ do total de trabalhos produzidos no GT2 do ENANCIB entre 2009 e 2014. Comparando com a Tabela 2 (Autores mais produtivos), observa-se que apenas duas das instituições mais produtivas não são as de origem dos pesquisadores mais produtivos: UNIRIO e UFSC. Observa-se ainda que as três primeiras instituições mais produtivas possuem a maior quantidade de autores mais produtivos, a saber: UFMG, UNESP e UFF, com cinco pesquisadores mais produtivos cada.

A Figura 4 apresenta a rede de coautoria entre as 40 instituições que publicaram trabalhos em colaboração científica no GT2 do ENANCIB entre 2009 e 2014. Portanto, das coautorias presentes no universo da pesquisa, as instituições foram apresentadas separadamente, considerando-se as suas distintas regiões de origem e suas características próprias.

Figura 4 - Representação gráfica da rede de colaboração institucional.

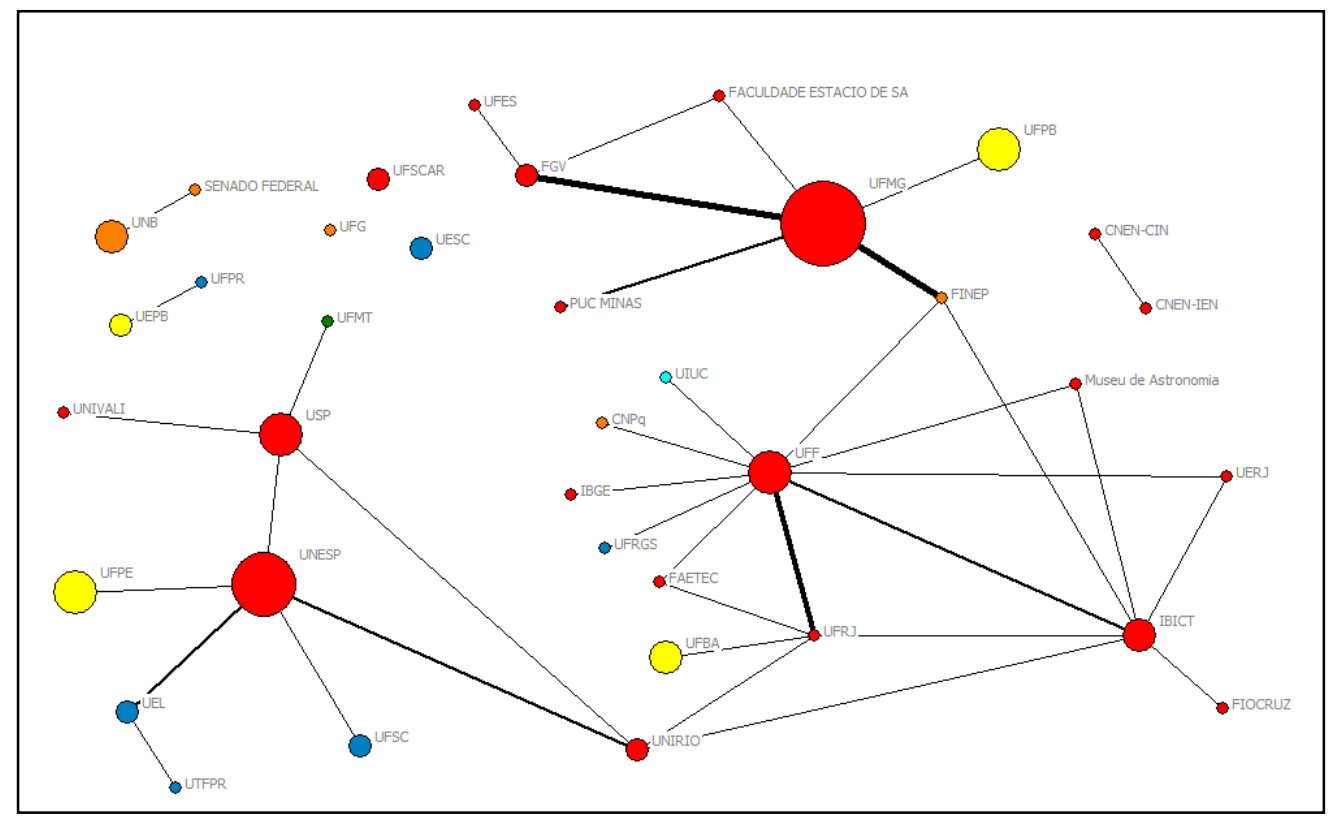

Fonte: Dados da pesquisa. 
A prática da colaboração científica se caracteriza como uma forma de unir esforços intelectual e material para o desenvolvimento dos trabalhos. Cabe esclarecer que as áreas dos círculos são proporcionais à frequência de coautorias dentro da própria instituição (colaboração intrainstitucional), e a espessura das linhas de retas é proporcional à intensidade de colaboração institucional (colaboração interinstitucional) (GRÁCIO; OLIVEIRA, 2011). Além disso, os círculos vermelhos referem-se às instituições da Região Sudeste do Brasil, os círculos em amarelo às da Região Nordeste, os círculos azuis à Região Sul, os círculos em laranja à Região Centro-Oeste e o círculo verde à instituição da Região Norte. Por sua vez, o azul claro representa instituições no âmbito do exterior.

De forma geral, há uma significativa participação de instituições da Região Sudeste, com destaque para a UFMG que lidera o ranking de instituição com mais colaboração interna. As Regiões Sul e Centro-Oeste têm cinco instituições cada, enquanto a Região Nordeste tem quatro instituições. Houve pouca participação da Região Norte, com apenas uma instituição.

Quanto à colaboração externa ou colaboração interinstitucional, observase que das 40 instituições que compõem a rede de colaboração científica, 27 $(44 \%)$ instituições formam o componente maior, cujas coautorias interinstitucionais mais intensas encontram-se entre a UFMG e FGV; UFMG e FINEP; UFRJ e UFF. As instituições que mais colaboraram nos trabalhos selecionados foram UFF, com 15 trabalhos, UFMG, com 10 trabalhos, FINEP com nove trabalhos, IBICT com oito, UNESP com sete trabalhos, UNIRIO com cinco trabalhos e USP e UFRJ com quatro trabalhos cada, seguidas da UEL com três e a FGV com dois.

Instituições como PUC MINAS, Museu de Astronomia, Faculdade Estácio de Sá, FAETEC, UERJ aparecem com duas colaborações externas e nenhuma colaboração interna. Outras instituições como UNB, UFSC, UFPE, UFPB, UEPB, UFBA aparecem com somente uma colaboração externa. 
No que tange à centralidade de grau, destaca-se a UFF, com 25\% do total das instituições que formam a rede em análise, seguida pelo IBICT, com 17,5\% e UFMG e UNESP, com 12,5\%, respectivamente.

No que se refere à colaboração interna ou colaboração intrainstitucional, a Figura 4 ainda reflete que a UFMG (36) é a instituição que se destaca em relação ao maior número de colaboração intrainstitucional, seguida pela UNESP (22), UFPB (12), UFF (9) e UFPE (10). Outras instituições como USP (7), UNB (7), UFRJ (6), UFSC (5), IBICT (5), UFBA (4) e UEL (3) também realizaram colaboração interna, mas em menor quantidade, seguidas por FGV, UNIRIO, UEPB, UFMT, CNEN-CIN, CNEN-IEN, UFPR, FIOCRUZ, UFRGS, IBGE, CNPQ, UFES, SENADO FEDERAL, UNIVALI e UTFPR, com apenas uma colaboração interna cada.

Observam-se ainda três conjuntos de díades, compostos por UNB e SENADO FEDERAL; UEPB e UFPR; CNEN-CIN e CNEN-IEN. Instituições como UFSCar (3), UESC (2) e UFG (1) apresentam-se isoladas, somente com coautoria intrainstitucional. Além disso, somente a UFF realizou uma colaboração internacional com a UIUC (Universidade de Illinois em UrbanaChampaign).

Outro ponto a ser destacado é que embora as quatro instituições sejam que mais realizam colaboração interna e também as mais produtivas, ou seja, UFMG, UNESP, UFF e USP, há pouco diálogo entre as três, com somente uma colaboração entre a UNESP e a USP.

No tocante às temáticas mais estudadas no GT2 do ENANCIB no período analisado, foram atribuídas 831 palavras-chave nos 215 trabalhos, sendo que 11 não apresentaram palavras-chave. Foi elaborada uma relação de palavras-chave atribuídas e ordenadas alfabeticamente. Em seguida, as palavraschave foram agrupadas por proximidade gramatical, considerando-se ainda singular/plural, o que possibilitou elaborar uma relação de 25 palavras-chave utilizadas em pelo menos cinco trabalhos (Tabela 5). 
Produção e colaboração científica em Organização e Representação do Conhecimento: análise bibliométrica do GT2 do ENANCIB no período de 2009 a 2014

Roberta Cristina Dal' Evedove Tartarotti, Mariângela Spotti Lopes Fujita

Tabela 5 - Temáticas mais abordadas.

\begin{tabular}{l|c}
\hline \multicolumn{1}{c|}{ PALAVRA-CHAVE } & Fi \\
\hline Indexação & \\
\hline Ontologia & 24 \\
\hline Organização da informação & 24 \\
\hline Organização do conhecimento & 20 \\
\hline Documento & 20 \\
\hline Recuperação da informação & 17 \\
\hline Representação da informação & 16 \\
\hline Modelagem & 14 \\
\hline Linguagem documental & 13 \\
\hline Classificação facetada de Ranganathan & 12 \\
\hline Ciência da Informação & 12 \\
\hline Folksonomia & 11 \\
\hline Semiótica & 11 \\
\hline Web & 10 \\
\hline Análise documental & 10 \\
\hline Arquivística & 9 \\
\hline Sistemas de Organização do Conhecimento & 8 \\
\hline Tesauro & 8 \\
\hline Análise de Domínio & 8 \\
\hline Fotografia & 7 \\
\hline Metadados & 7 \\
\hline Sistemas de recuperação da informação & 7 \\
\hline Organização e representação do conhecimento & 6 \\
\hline Taxonomia & 5 \\
\hline Classificação bibliográfica & \\
\hline
\end{tabular}

Fonte: Dados da pesquisa.

De forma geral, o campo da Organização e Representação do Conhecimento apresenta três grandes tendências de pesquisa: Tecnológica: volta-se para as questões em torno das novas perspectivas e ferramentas de aplicação; Cultural: como tais questões da área se colocam mediante o contexto social, ou seja, quais desdobramentos surgem nesse processo mediador da cultura de quem produz, quem organiza, incluindo os problemas éticos decorrentes deste processo; e Epistemológica: o olhar recai sobre como a área se configura como um domínio, onde incide uma preocupação em torno de seus fundamentos teórico-metodológicos.

Por meio da incidência das palavras-chave, verifica-se que as mais utilizadas foram: indexação e ontologia, seguidas por organização da informação e organização do conhecimento. Considerando-se ainda as demais temáticas abordadas, verifica-se uma maior concentração nas abordagens 
tecnológica e epistemológica e poucos trabalhos em torno da abordagem cultural do campo da Organização e Representação do Conhecimento neste universo de pesquisa.

\section{Considerações finais e recomendações}

Por meio dos dados coletados e sua respectiva análise, foi possível realizar um mapeamento da produção científica na área de Organização e Representação do Conhecimento nos anais do GT2 do ENANCIB entre 2009 e 2014. Ao identificarmos as principais características quanto ao perfil dos atores que atuam na produção científica desta temática, buscamos contribuir para um panorama e avaliação da consistência da área.

Um ponto a ser observado é a maior parte dos trabalhos ter sido produzida em colaboração científica tanto interinstitucional como intrainstitucional, destacando-se a colaboração orientador/orientando. Além disso, considera-se relevante uma maior colaboração entre os pesquisadores com interesses em temas correlatos, porém, de diferentes instituições, possibilitando maior interação e compartilhamento das pesquisas e fortalecimento da área de Organização e Representação do Conhecimento tanto no cenário brasileiro como internacional.

Aponta-se a necessidade de maior padronização nas súmulas dos trabalhos do ENANCIB para os próximos anos, tornando a coleta mais rápida e, consequentemente, a análise dos dados obtidos, colaborando para o interesse de mais pesquisadores na abordagem bibliométrica, tão relevante para o mapeamento da produção e comunicação científica na contemporaneidade.

Como recomendação para desdobramentos de futuras pesquisas, sugerese que a análise da produção científica em Organização e Representação do Conhecimento seja ampliada para outros universos e aplicada com outras variáveis. Com base nisso, para se obter um panorama completo da temática, entre elas a análise de citações e cocitações e de coocorrência de temáticas (utilizando-se as palavras-chave), é necessário evidenciar a frente de pesquisa e o referencial teórico adotado por sua comunidade científica, contribuindo ainda 


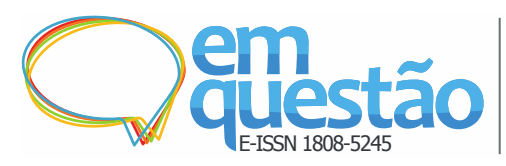

Produção e colaboração científica em Organização e Representação do Conhecimento: análise bibliométrica do GT2 do ENANCIB no período de 2009 a 2014

Roberta Cristina Dal'Evedove Tartarotti, Mariângela Spotti Lopes Fujita

mais com o desenvolvimento do saber científico nesta temática. Ademais, acredita-se que a análise dos indicadores específicos das redes (de densidade, centralidade de grau, centralidade de intermediação e centralidade de proximidade), poderá revelar, de forma mais detalhada, o papel desempenhado pelos pesquisadores em suas posições de proximidade e intermediação nas redes aqui apresentadas.

Em suma, ressalta-se a importância da análise das relações dialógicas entre os pesquisadores e instituições que compõem o universo científico da Organização e Representação do Conhecimento, disponibilizando-as para análise e reflexão, por meio das abordagens bibliométricas.

\section{Referências}

ABBAGNANO, N. Dicionário de filosofia. Martins Fontes: São Paulo, 2000.

ASSOCIAÇÃO NACIONAL DE PESQUISA E PÓS-GRADUAÇÃO EM CIÊNCIA DA INFORMAÇÃO (ANCIB). Estatuto. Rio de Janeiro, 2010.

BOURDIEU, P. O campo científico. In: ORTIZ, R. (Org.). Pierre Bourdieu: sociologia. São Paulo: Ática, 1983. p. 122-155. (Coleção Grandes Cientistas Sociais; 39).

CARRIZO SAINEO, G. Hacia un concepto de bibliometría. Revista de Investigación Iberoamericana en Ciencia de la Información y Documentación, [S.1.], v. 1, n. 2, p. 1-10, jul.Idec. 2000.

CURRÁS, E. Integración vertical de las ciências aplicada a redes sociales sociedad de la información en sus relaciones sistémicas. In: POBLACIÓN, D. A.; MUGNAINI, R.; RAMOS, L. M. S. V. C. (Orgs.). Redes sociais e colaborativas: em informação científica. São Paulo: Angellara, 2009. p. 57-92.

DAHLBERG, I. ISKO and Knowledge Organization's 25th anniversary: the future of Knowledge Organization and ISKO painel discussion. Knowledge Organization, Würzburg, v. 41, n. 4, p. 327-331, 2014.

DAL'EVEDOVE, P. R.; FUJITA, M. S. L.; TARTAROTTI, R. C. D. E. A produção científica periódica na temática indexação: análise bibliométrica no período de 2003 a 2012. In: ENCONTRO NACIONAL DE PESQUISA EM CIÊNCIA DA INFORMAÇÃO, 14., 2013, Florianópolis. Anais eletrônicos... Florianópolis: UFSC, 2013. Disponível em:

<http://enancib.ibict.br/index.php/enancib/xivenancib/paper/view/4358/3481>. Acesso em: 21 set. 2015. 
FUJINO, A. et al. Políticas públicas de incentivo à formação de redes sociais e colaborativas em ciência e tecnologia. In: POBLACIÓN, D. A.; MUGNAINI, R.; RAMOS, L. M. S. V. C. (Orgs.). Redes sociais e colaborativas: em informação científica. São Paulo: Angellara, 2009. p. 205-237.

FUNARO, V. M. B. de O. et al. Redes colaborativas entre autores de revistas cientificas em Odontologia e Medicina. In: POBLACIÓN, D. A.; MUGNAINI, R.; RAMOS, L. M. S. V. C. (Orgs.). Redes sociais e colaborativas: em informação científica. São Paulo: Angellara, 2009. p. 347-377.

GRÁCIO, M. C. C.; OLIVEIRA, E. F. T. de. Produção e comunicação da informação em C\&I - GT7 da ANCIB: análise bibliométrica no período de 2003/2009. Liinc em Revista, Rio de Janeiro, v. 7, n. 1, p. 248-263, mar. 2011.

KATZ, J. S.; MARTIN, B. R. What is research collaboration? Research Policy, v. 26, p. 1-18, 1997.

MACIAS-CHAPULA, C. A. O papel da informetria e da cienciometria e sua perspectiva nacional e internacional. Ciência da Informação, Brasília, v. 27, n. 2, p. 134-140, 1998.

MAI, J.-E. Analysis in indexing: document and domain centered approaches.

Information Processing and Management, [S.1.], v. 41, n. 3, p. 599-611, 2005.

MALTRÁS BARBA, B. Los indicadores bibliométricos: fundamentos y aplicación al análisis de la ciencia. Gijón: Trea, 2003.

MARCELO, J. F., HAYASHI, M. C. P. I. Estudo bibliométrico sobre a produção científica no campo da Sociologia da Ciência. Informação \& Informação, Londrina, v. 18, n. 3, p. 138-153, 2013.

MEADOWS, A. J. A comunicação científica. Brasília: Briquet de Lemos, 1999.

OKUBO, Y. Bibliometric indicators and analysis of research systems: methods and examples. Paris: OECD, 1997.

OLIVEIRA, E. F. T. de; GRÁCIO, M. C. C.; SILVA, A. C. C. da.

Investigadores con mayor visibilidad en Organización y Representación del Conocimiento. Scire, Zaragoza, v. 16, n. 2, p. 39-45, 2010.

PINTO, A. L. et al. Visualização da informação das redes sociais através de programas de cienciografia. In: POBLACIÓN, D. A.; MUGNAINI, R.; RAMOS, L. M. S. V. C. (Orgs.). Redes sociais e colaborativas: em informação científica. São Paulo: Angellara, 2009. p. 289-312.

POBLACIÓN, D. A.; MUGNAINI, R.; RAMOS, L. M. S. V. C. (Orgs.). Redes sociais e colaborativas: em informação científica. São Paulo: Angellara, 2009. 
PORTAL DA ASSOCIAÇÃO NACIONAL DE PESQUISA E PÓSGRADUAÇÃO EM CIÊNCIA DA INFORMAÇÃO (ANCIB). 2015. Disponível em: 〈http://www.ancib.org.br〉. Acesso em: 22 nov. 2015.

TEIXEIRA, D. S. Pesquisa, desenvolvimento experimental e inovação industrial: motivações da empresa privada e incentivos do setor público. In: MARCOVITCH, J. Administração em ciência e tecnologia. São Paulo: Edgard Blucher, 1983, p. 43-91.

SIQUEIRA, J. C. Biblioteconomia, Documentação e Ciência da informação: história, sociedade, tecnologia e pós-modernidade. Perspectivas em Ciência da Informação, Belo Horizonte, v. 15, n. 3, p. 52-66, set./dez. 2010.

SMIRAGLIA, R. P. Domain coherence within Knowledge Organization: people, interacting theoretically, across geopolitical and cultural boundaries. In: Annual CAIS/ACSI Conference, 39., June 2-4, 2011, Canada. Proceedings... Canada: University of New Brunswick, 2011. p. 1-6.

SPINAK, E. Dicionário enciclopédico de Bibliometria, Cienciometria e Informetria. Venezuela: UNESCO, 1996.

SPINAK, E. Indicadores cienciométricos. Ciência da Informação, Brasília, v. 27, n. 2, p. 141-148, 1998.

VANZ, S. A. S; CAREGNATO, S. E. Estudos de citação: uma ferramenta para entender a comunicação científica. Em Questão, Porto Alegre, v. 9, n. 2, p. 295 307, 2003.

\title{
Production and scientific collaboration in Organization and Representation of Knowledge: a bibliometric analysis of GT2 of ENANCIB in the period from 2009 to 2014
}

\begin{abstract}
This study aimed to characterize the Brazilian scientific community in Organization and Representation of Knowledge area of the Information Science, from the publication of proceedings papers in GT2 of the ENANCIB (Encontro Nacional de Pesquisa em Ciência da Informação) from 2009 to 2014 through production and connection bibliometric indicators. From the 215 proceedings papers were analyzed the most productive authors, gender relations among authors, type of authorship, the most productive institutions, the most studied themes and scientific collaboration networks among authors/institutions, built using Excel and UCINET softwares. The results indicate 27 researchers and 14 institutions as the most productive ones, especially in the Southeast Region of Brazil. Most of the proceedings papers were performed by female
\end{abstract}


authors and in scientific collaboration, especially in intra-institutional collaboration and the largest collaborative network constituted by 37 authors. There was greater focus on technological and epistemological approaches and few proceedings papers about the cultural approach to the Organization and Knowledge Representation field as their universe.

Keywords: Organization and Representation of Knowledge. Scientific production. Bibliometric studies. Scientific collaboration. ENANCIB.

Recebido: 29/04/2016

Aceito: $30 / 06 / 2016$

${ }^{1}$ De acordo com MAI (2005, p. 605), o conceito de domínio pode ser entendido como uma área do conhecimento, um corpo de literatura ou um grupo de atores trabalhando juntos em uma organização ou que compartilham objetivos comuns.

${ }^{2}$ Questões em Rede. Disponível em: 〈http://www.questoesemrede.uff.br/index.php/repositorio〉. Acesso em: 22 nov. 2015. 